\title{
Novel Butterfly Tungsten-Osmium Carbido Cluster Complexes from the Reaction of $\mathrm{Os}_{3}(\mathrm{CO})_{10}(\mathrm{NCMe})_{2}$ with $\mathrm{CpW}(\mathrm{CO})_{3}\left(\mathrm{CH}_{2} \mathrm{SMe}\right)$
}

\author{
Jia-Huey Gong,' Chyuan-Wu Tsay,' Wen-Cheng Tu, Yun Chi, 1.3 \\ Shie-Ming Peng, ${ }^{2}$ and Gene-Hsiang Lee $^{2}$
}

Received January 31, 1995

Condensation of the triosmium acetonitrile complex $\mathrm{Os}_{3}(\mathrm{CO})_{10}(\mathrm{NCMe})_{2}$ with the sulfido complex $\mathrm{CpW}(\mathrm{CO})_{3}\left(\mathrm{CH}_{2} \mathrm{SMe}\right)$ in refluxing $\mathrm{THF}$ solution produced three sulfur-containing compounds $\mathrm{Os}_{3}(\mathrm{CO})_{10}(\mu-\mathrm{H})(\mu-\mathrm{SMe})(1), \mathrm{Os}_{3}(\mathrm{CO})_{11}$ $\left[\mathrm{S}(\mathrm{Me}) \mathrm{CH}_{2} \mathrm{~W}(\mathrm{CO})_{3} \mathrm{Cp}\right]$ (2) and $\mathrm{CpWO}_{3}(\mathrm{CO})_{12}\left(\mu-\mathrm{CH}_{2}\right)(\mu t-\mathrm{SMe})$ (3). Clusters 2 and 3 were products involving a $1: 1$ combination of starting materials and were characterized by $\mathrm{X}$-ray diffraction studies. Crystals of 2 belongs to monoclinic space group $\mathrm{P} 2 / \mathrm{c}$ with $a=8.418(2), b=11.912(2)$, $c=28.288(4)$ A. $\beta=97.64(2)^{\circ}, Z=4 ; R_{f}=0.048, R_{w}=0.044$. Crystal dara for 3: space group $\mathrm{P} 2_{1} / c, a=18.156(4), b=9.255(6), c=15.347(4) A, \beta=103.49(2)^{\circ}$, $Z=4 ; R_{F}=0.047, R_{W}=0.045$. Upon thermolysis in toluene, the methylene cluster 3 released $\mathrm{CO}$ and induced $\mathrm{C}-\mathrm{H}$ bond activation to afford two tetrametallic carbido clusters with formula $\mathrm{CpWO}_{3}(\mathrm{CO})_{9}\left(\mu_{4}-\mathrm{C}\right)(\mu-\mathrm{H})_{2}(\mu$-SMe $)(4)$ and $\mathrm{CpWOs}_{3}(\mathrm{CO})_{11}\left(\mu_{4}-\mathrm{C}\right)(\mu$-SMe $)(5)$ as the principle products. The first complex possesses a butterfly framework encapsulating a $\mu_{4}$-C ligand and a $\mu^{-\mathrm{SMe}}$ ligand linking a $\mathrm{W}-\mathrm{O}$ s edge, whereas the second product adopts a puckered, cyclic arrangement of $\mathrm{WOs}_{3}$ metal atoms with $\mu$-SMe ligand located on a nonbonding Os-Os vector. Complex 4 crystallizes in monoclinic space group $\mathrm{P} 2 / \mathrm{c}$ with $a=15.633(4) \AA, \quad b=8.699(3) \AA, \quad c=15.422(4) \AA, \quad \beta=93.12(2)^{\circ}, \quad Z=4$, $R=0.036, R_{W}=0.034$ for 2780 observed reflctions. Crystal data for 5 : space group $P$ mat $a=14.542(3), b=13.710(6), c=11.758(3) \AA, Z=4, R_{F}=0.038$, $R_{w}=0.037$ for 1826 observed reflections. A variable temperature ${ }^{1} \mathrm{H}$ NMR study was also presented to demonstrate the solution fluxionality of 5 .

KEY WORDS: Osmium; tungsten; thiolato; methylene; carbide; heterometallic clusters.

\footnotetext{
'Department of Chemistry. National Tsing Hua University, Hsinchu 300, Taiwan.

-Department of Chemistry, National Taiwan University. Taipei 107. Taiwan.

"To whom all correspondence should be addressed.
} 


\section{INTRODUCTION}

The chemistry of mixed-metal cluster complexes has been the subject of intensive research for many years [1], due to the potential of these systems in studying the bonding and reactivity of organic ligands by varying the electronic and steric properties of transition-metal elements. With the objective to find a systematic method for the preparation of heterometallic cluster complexes, our research group has examined the direct condensation of group 6 hydride, acetylide and aldehyde complexes, $L W(C O){ }_{3} \mathrm{H}$, $L W(\mathrm{CO})_{3}(\mathrm{CCPh})$, and $L W(\mathrm{CO})_{3}\left(\mathrm{CH}_{2} \mathrm{CHO}\right), L=\mathrm{Cp}$ and $\mathrm{Cp}^{*}$, and group 8 cluster compounds, such as $\mathrm{Os}_{3}(\mathrm{O})_{10}(\mathrm{NCMe})_{2}$ [2], $\mathrm{Ru}_{3}(\mathrm{O})_{12}$ [3], $\mathrm{Ru}_{3}(\mathrm{CO})_{10}(\mu-\mathrm{H})(\mu$-COMe $)$ [4], $\mathrm{Ru}_{3}(\mathrm{CO})_{10}\left(\mu_{3}-\mathrm{NPh}\right)$ [5], and $\mathrm{Ru}_{3}(\mathrm{CO})_{10}(\mu-\mathrm{H})\left(\mu-\mathrm{PPh}_{2}\right)$ [6]. As a result, we obtained a series of heterometallic cluster compounds containing a variety of hydrocarbyl fragments and ligands which possess oxygen, nitrogen, or phosphorus substituents. In order to further extend these cluster assembling reactions to cluster compounds with sulfur-containing hydrocarbon ligands, we have carried out the reactions of osmium thiolato complex $\mathrm{Os}_{3}(\mathrm{CO})_{10}(\mu-\mathrm{H})$ $\left(\mu\right.$-SMe) with $\mathrm{CpW}(\mathrm{CO})_{3} \mathrm{H}[7]$ and the reaction of $\mathrm{Os}_{3}(\mathrm{CO})_{10}(\mathrm{NCMe})_{2}$ with $\mathrm{CpW}(\mathrm{CO})_{3}\left(\mathrm{CH}_{2} \mathrm{SMe}\right)$. In this report, we describe the spectroscopic and structural characterization of two $\mathrm{WOs}_{3}$ thiolate clusters obtained from the second reaction, and the X-ray structures of two heterometallic carbido complexes prepared from the subsequent thermolysis of the cluster $\mathrm{CpWOs}_{3}(\mathrm{CO})_{12}\left(\mu-\mathrm{CH}_{2}\right)(\mu$-SMe $)$ through a consecutive sequence of double $\mathrm{C}-\mathrm{H}$ bond activation and formation of carbide ligand.

\section{EXPERIMENTAL PROCEDURE}

General Information and Materials. Infrared spectra were recorded on a Perkin Elmer 2000 FT-IR spectrometer. ${ }^{1} \mathrm{H}$ and ${ }^{13} \mathrm{C}$ NMR spectra were recorded on a Bruker AM-400, a Varian Gemini-300 or a Varian Unity400 instrument. Chemical shifts are quoted with respect to internal standard tetramethylsilane $\left({ }^{1} \mathrm{H}\right.$ and $\left.{ }^{13} \mathrm{C} N \mathrm{NM}\right)$. Mass spectra were obtained on a JEOL-HX110 instrument operating in the fast atom bombardment $(\mathrm{FAB})$ mode. $\mathrm{CpW}(\mathrm{CO})_{3}\left(\mathrm{CH}_{2} \mathrm{SMe}\right)$ was prepared from the reaction between $\left[\mathrm{CpW}(\mathrm{CO})_{3}\right][\mathrm{Na}]$ and $\mathrm{MeSCH}_{2} \mathrm{Cl}$ according to the literature method [8]. All reactions were performed under a nitrogen atmosphere using deoxygenated solvents dried with an appropriate reagent. The progress of reactions was monitored by analytical thin-layer chromatography (5735 Kieselgel $60 \mathrm{~F}_{254}$, E. Merck) and the products were separated on commercially available preparative thin-layer 
chromatographic plates (Kieselgel $60 \mathrm{~F}_{254}$, E. Merck). Elemental analyses were performed at the NSC Regional Instrumentation Center at National Cheng Kung University, Tainan, Taiwan.

Reaction of $\mathrm{Os}_{3}(\mathrm{CO})_{10}(\mathrm{NCMe})_{2}$ and $\mathrm{CpW}(\mathrm{CO})_{2}\left(\eta^{2}-\mathrm{CH}_{2} \mathrm{SMe}\right)$. A THF solution $(30 \mathrm{~mL})$ of $\mathrm{Os}_{3}(\mathrm{CO})_{10}(\mathrm{NCMe})_{2}(212 \mathrm{mg}, 0.228 \mathrm{mmol})$ and $\mathrm{CpW}(\mathrm{CO})_{3}\left(\mathrm{CH}_{2} \mathrm{SMe}\right)(100 \mathrm{mg}, 0.273 \mathrm{mmol})$ was heated to reflux for 2 hours, during which the color changed from orange to red-orange. After cooling the solution to room temperature, the solvent was evaporated and the residue was separated by thin layer chromatography (dichloromethane: hexane $=1: 3$ ), giving $84 \mathrm{mg}$ of light-yellow $\mathrm{Os}_{3}(\mathrm{CO})_{10}(\mu-\mathrm{H})(\mu-\mathrm{SMe})(1,0.094 \mathrm{mmol}, 41 \%), 49 \mathrm{mg}$ of yellow-orange $\mathrm{Os}_{3}(\mathrm{O})_{11}\left[\mathrm{~S}(\mathrm{Me}) \mathrm{CH}_{2} \mathrm{~W}(\mathrm{CO})_{3} \mathrm{Cp}\right](2,0.039 \mathrm{mmol}, 19 \%)$ and $22 \mathrm{mg}$ of orange-red $\mathrm{CpWOs}_{3}(\mathrm{CO})_{12}\left(\mu-\mathrm{CH}_{2}\right)(\mu-\mathrm{SMe})(3,0.018 \mathrm{mmol}, 8 \%)$. Single crystals of 2 and 3 suitable for $\mathrm{X}$-ray analysis were obtained from a layered solution of dichloromethane-heptane at room temperature.

Spectral data for 1: MS (FAB, $\left.{ }^{192} \mathrm{Os}\right), m / z 904\left(\mathrm{M}^{+}\right)$. IR $\left(\mathrm{C}_{6} \mathrm{H}_{12}\right)$ : v(CO), 2108(m), 2066(vs), 2058(vs), 2034(w), 2023(vs), 2018(vs), 1998(vs), 1989(vs), 1963(s), 1955(vw) cm-1; 'H NMR $\left(\mathrm{CDCl}_{3}, 294 \mathrm{~K}\right): \delta 2.43$ $(s, 3 \mathrm{H}),-17.16(s, 1 \mathrm{H}) ;{ }^{13} \mathrm{C} \mathrm{NMR}\left(\mathrm{CDCl}_{3}, 294 \mathrm{~K}\right): \mathrm{CO}, \delta 180.8,180.2$, $176.3(2 \mathrm{C}), 173.7(2 \mathrm{C}), 170.7(2 \mathrm{C}), 169.2(2 \mathrm{C}) ; \delta 38.7\left(\mathrm{SCH}_{3}\right)$. Anal. Calcd for $\mathrm{C}_{11} \mathrm{H}_{4} \mathrm{O}_{10} \mathrm{~S}_{1} \mathrm{Os}_{3}$ : C, 14.60; H, 0.45 Found: $\mathrm{C}, 14.76 ; \mathrm{H}, 0.47$.

Spectral data for 2: MS (FAB, $\left.{ }^{192} \mathrm{Os},{ }^{184} \mathrm{~W}\right), m / z 1278\left(\mathrm{M}^{+}\right)$. IR $\left(\mathrm{C}_{6} \mathrm{H}_{12}\right): \quad v(\mathrm{CO}), 2105(\mathrm{vw}), 2068(\mathrm{vw}), 2051(\mathrm{~s}), 2031(\mathrm{~s}), 2017(\mathrm{vs})$, 1999(w), 1988(w), 1975(w), 1959(w), 1950(m), 1941(w), 1932(w) $\mathrm{cm}^{-1}$; 'H NMR $\left(\mathrm{CDCl}_{3}, 294 \mathrm{~K}\right): \delta 5.52(s, 5 \mathrm{H}), 2.98(s, 2 \mathrm{H}), 2.81(s, 3 \mathrm{H})$, ${ }^{13} \mathrm{CNMR}\left(\mathrm{CD}_{2} \mathrm{Cl}_{2}, 230 \mathrm{~K}\right): \mathrm{CO}, \delta 225.6\left(J_{W-C}=143 \mathrm{~Hz}\right), 217.3(2 \mathrm{C}$, $\left.J_{\| \cdot C}=154 \mathrm{~Hz}\right), 194.7\left(d, J_{C-C}=36.5 \mathrm{~Hz}\right), 185.5\left(d, J_{C \cdot C}=36.5 \mathrm{~Hz}\right), 185.5$ (2C), $183.5(2 \mathrm{C}), 177.0,176.3,171.8,171.3,170.8 ; \delta 92.3\left(\mathrm{C}_{5} \mathrm{H}_{5}\right), 34.1$ $\left(\mathrm{CH}_{3}\right), 3.5\left(\mathrm{CH}_{2}, J_{W-C}=47 \mathrm{~Hz}\right)$. Anal. Calcd for $\mathrm{C}_{21} \mathrm{H}_{10} \mathrm{O}_{14} \mathrm{~S}_{1} \mathrm{Os}_{3} \mathrm{~W}_{1}: \mathrm{C}$, 19.82; H, 0.79 Found: C, 19.66; H, 0.77.

Spectral data for 3: MS (FAB, $\left.{ }^{192} \mathrm{Os},{ }^{184} \mathrm{~W}\right), m / z 1222\left(\mathrm{M}^{+}\right)$. $1 \mathrm{R}\left(\mathrm{C}_{6} \mathrm{H}_{12}\right): \quad v(\mathrm{CO}), 2109(\mathrm{w}), 2063(\mathrm{~s}), 2029(\mathrm{vs}), 2023(\mathrm{vs}), 2008(\mathrm{vs})$, 1982(w), 1948(w), cm ${ }^{-1}$; ${ }^{1} \mathrm{H}$ NMR $\left(\mathrm{CDCl}_{3}, 294 \mathrm{~K}\right): \delta 5.47(s, 5 \mathrm{H}), 3.84$ $\left(d, 1 \mathrm{H},{ }^{2} J_{H-H}=4.0 \mathrm{~Hz}\right), 3.41 \quad\left(d, 1 \mathrm{H},{ }^{2} J_{H-H}=4.0 \mathrm{~Hz}\right), 2.19(s, 3 \mathrm{H})$; ${ }^{17} \mathrm{C} N M R\left(\mathrm{CDCl}_{3}, 294 \mathrm{~K}\right): \mathrm{CO}, \delta 222.0\left(J_{w-C}=141 \mathrm{~Hz}\right), 215.2\left(J_{w-C}=\right.$ $106 \mathrm{~Hz}), 187.2,185.1,183.8(4 \mathrm{C}), 174.1 \quad(2 \mathrm{C}), 167.8(2 \mathrm{C}$, br $) ; \delta 88.9$ $\left(\mathrm{C}_{5} \mathrm{H}_{5}\right), \quad 34.7\left(\mathrm{CH}_{2}, \quad J_{W-C}=36 \mathrm{~Hz}\right), 27.9\left(\mathrm{CH}_{3}\right)$. Anal. Calcd for $\mathrm{C}_{19} \mathrm{H}_{10} \mathrm{O}_{12} \mathrm{~S}_{1} \mathrm{Os}_{3} \mathrm{~W}_{1}$ : C, 18.76; H, 0.83 Found: C, 18.94; H, 0.85 .

Thermolysis of 3. A toluene solution $(15 \mathrm{~mL})$ of $3(25 \mathrm{mg}$, $0.021 \mathrm{mmol}$ ) was heated to reflux for 1.5 hours, during which the color 
changed from red-orange to orange-brown. After allowing the solution to cool to room temperature, the solvent was evaporated and the residue was separated by thin layer chromatography (dichloromethane: hexane $=1: 2$ ), giving $7 \mathrm{mg}$ of green $\mathrm{CpWOs}_{3}(\mathrm{CO})_{9}\left(\mu_{4}-\mathrm{C}\right)(\mu-\mathrm{H})_{2}(\mu-\mathrm{SMe})(4,0.006 \mathrm{mmol}$, $30 \%)$ and $9 \mathrm{mg}$ of orange $\mathrm{CpWOs}_{3}(\mathrm{CO})_{11}\left(\mu_{4}-\mathrm{C}\right)(\mu-\mathrm{SMe})(5,0.007 \mathrm{mmol}$, $37 \%$ ). Single-crystals of $\mathbf{4}$ suitable for $\mathrm{X}$-ray analysis were obtained from a layered solution of dichloromethane-heptane at room temperature, whereas crystals of 5 was obtained from a mixture of chloroform-heptane.

Spectral data for 4: MS (FAB, $\left.{ }^{192} \mathrm{Os},{ }^{184} \mathrm{~W}\right), m / z 1138\left(\mathrm{M}^{+}\right)$. IR $\left(\mathrm{C}_{6} \mathrm{H}_{12}\right): v(\mathrm{CO}), 2087$ (s), 2057(vs), 2044(w), 2034(vs), 2012(vs), 2007 $(s, \mathrm{sh}), 1988(\mathrm{~s}), 1974(\mathrm{w}), 1876(\mathrm{~m}) \mathrm{cm}^{-1} ;{ }^{1} \mathrm{H} \mathrm{NMR}\left(\mathrm{CDCl}_{3}, 294 \mathrm{~K}\right): \delta 5.46$ $(s, 5 \mathrm{H}), 2.76(s, 3 \mathrm{H}), \quad-17.04 \quad(s, 1 \mathrm{H}), \quad-24.14 \quad(s, 1 \mathrm{H}) ;{ }^{13} \mathrm{C} \mathrm{NMR}$ $\left(\mathrm{CDCl}_{3}, 294 \mathrm{~K}\right): \mathrm{CO}, \delta 220.4\left(J_{\|-C}=179 \mathrm{~Hz}\right), 181.3,179.8,176.6,173.9$, $172.6,169.4,165.8,165.6 ; \delta 305.4\left(\mu_{4}-\mathrm{C}\right), 91.3\left(\mathrm{C}_{5} \mathrm{H}_{5}\right), 36.2\left(\mathrm{CH}_{3}\right)$. Anal. Calcd for $\mathrm{C}_{16} \mathrm{H}_{10} \mathrm{O}_{9} \mathrm{~S}_{1} \mathrm{Os}_{3} \mathrm{~W}_{1}$ : C, 16.97; H, 0.89. Found: C, 16.80; H, 0.91.

Spectral data for 5: MS (FAB, $\left.{ }^{192} \mathrm{Os},{ }^{184} \mathrm{~W}\right), m / z 1192\left(\mathrm{M}^{+}\right)$. IR $\left(\mathrm{C}_{6} \mathrm{H}_{12}\right): v(\mathrm{CO}), 2084(\mathrm{vw}), 2060(\mathrm{vs}), 2053(\mathrm{~m}, \mathrm{sh}), 2042(\mathrm{vs}), 2033(\mathrm{w})$, $2017(\mathrm{~m}), \quad 2000(\mathrm{vs}), \quad 1976(\mathrm{w}), \quad 1963(\mathrm{~m}), \quad 1929(\mathrm{vw}), \quad 1917(\mathrm{w}), 1877(\mathrm{vw})$, $1868(\mathrm{vw}) \mathrm{cm}^{-1} .{ }^{1} \mathrm{H} \mathrm{NMR}\left(\mathrm{CD}_{2} \mathrm{Cl}_{2}, 218 \mathrm{~K}\right): \mathrm{C}_{5} \mathrm{H}_{5}: \delta 5.81,5.47,5.40$; $\mathrm{CH}_{3}: \delta 1.91,1.89,1.83 .{ }^{13} \mathrm{C} \mathrm{NMR}\left(\mathrm{CD}_{2} \mathrm{Cl}_{2}, 230 \mathrm{~K}\right): \mu_{4}-\mathrm{C}: \delta 329.9,329.7$, $316.1 ; \quad \mathrm{W}-\mathrm{CO}: \delta 224.0 \quad\left(J_{W-C}=161 \mathrm{~Hz}\right), 219.4 \quad\left(J_{W-C}=161 \mathrm{~Hz}\right), \quad 208.0$ $\left(J_{n: C}=162 \mathrm{~Hz}\right) ; \quad \mathrm{C}_{5} \mathrm{H}_{5}: \quad \delta 92.5,91.7,86.9 ; \quad \mathrm{CH}_{3}: \delta 33.7, \quad 32.6, \quad 31.5$. Anal. Calcd for $\mathrm{C}_{18} \mathrm{H}_{8} \mathrm{O}_{11} \mathrm{~S}_{1} \mathrm{Os}_{3} \mathrm{~W}_{1}: \mathrm{C}, 18.22 ; \mathrm{H}, 0.68$. Found: $\mathrm{C}, 18.13$; $\mathrm{H}, 0.66$.

$X$-Ray Crystallography. Diffraction measurements for all complexes were carried out on a Nonius CAD-4 diffractometer. All reflections were corrected for Lorentz, polarization, and absorption effects. Data reduction and refinement were performed using the NRCC-SDP-VAX packages. Empirical absorption corrections were applied by using $\Psi$ scan data. Anisotropic thermal parameters were introduced for all nonhydrogen atoms. The hydrogen atoms were assumed to attach at the idealized position. The hydrogen atom positions were included in the structure factor calculations but not refined in the final least squares cycles. The combined data collection and refinement parameters are summarized in Table I. Atomic positional parameters for $\mathbf{2}, \mathbf{3}, \mathbf{4}$, and $\mathbf{5}$ are presented in Tables II-V, whereas selected bond angles and lengths are listed in Tables VI-IX, respectively.

Lattice parameters of 2 were determined from 25 randomly selected high angle reflections with $2 \theta$ angles in the range $18.8-24.8^{\circ}$. The minimum and maximum transmission factors were 0.35 and 1.00 , respectively. Full matrix least-squares refinement with 50 atoms and 362 parameters gave 


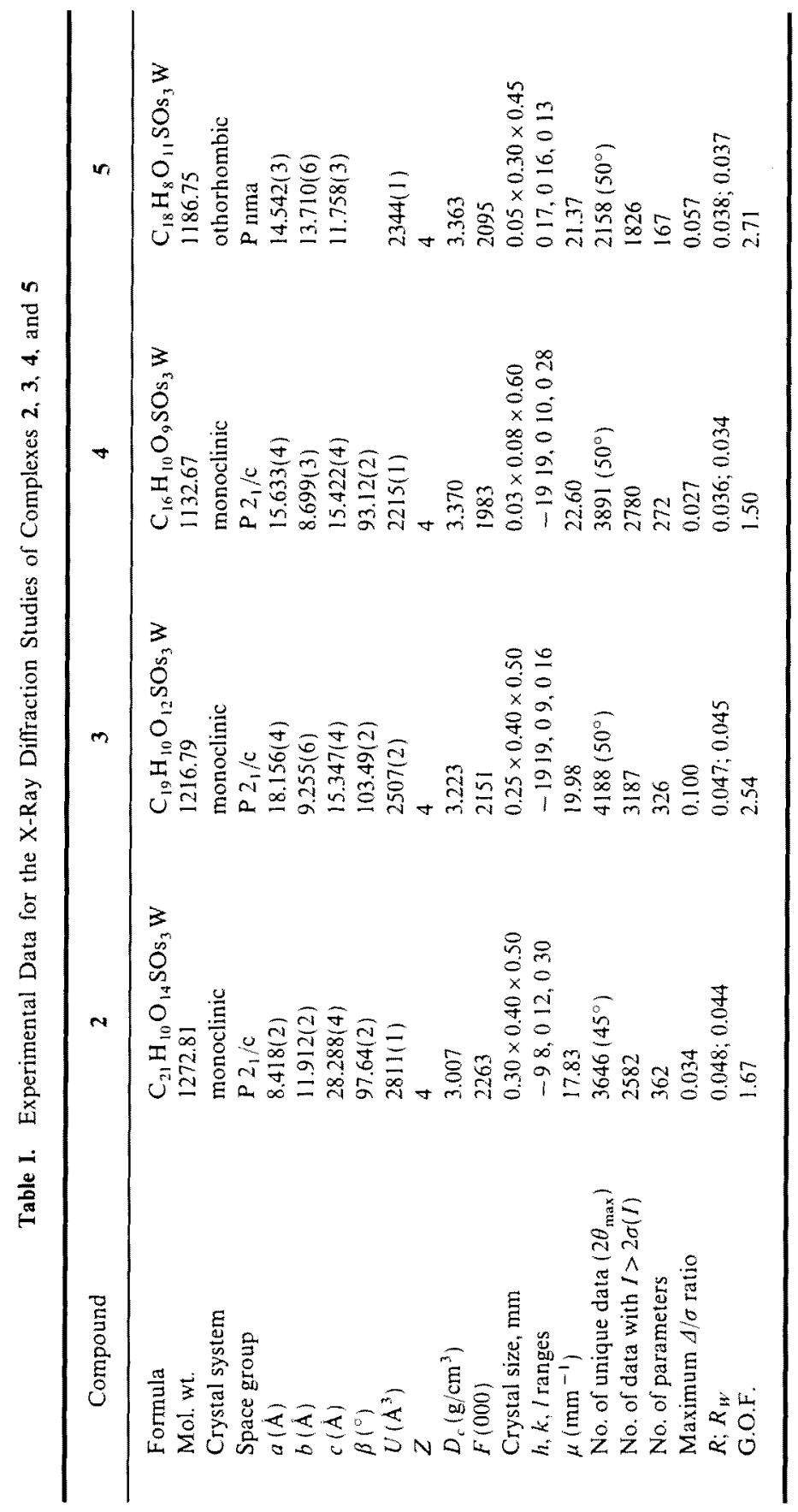


Table II. Atomic Coordinates and Equivalent Isotropic Displacement Coefïients for 2

\begin{tabular}{|c|c|c|c|c|}
\hline & $x$ & $y$ & $=$ & $B(\mathrm{eq})^{\prime \prime}$ \\
\hline Os! & $0.83645(12)$ & $0.70043(10)$ & $0.15290(3)$ & $2.72(4)$ \\
\hline Os2 & $1.01813(13)$ & $0.85300(10)$ & $0.10413(3)$ & $3.29(5)$ \\
\hline Os 3 & $0.85147(13)$ & $0.67031(10)$ & $0.05206(3)$ & $3.43(5)$ \\
\hline w & $0.61407(12)$ & $0.25076(10)$ & $0.16180(3)$ & $2.75(4)$ \\
\hline$S$ & $0.6501(7)$ & $0.5491(6)$ & $0.15608(19)$ & $3.0(3)$ \\
\hline $\mathrm{Cl}$ & $1.018(3)$ & $0.6031(24)$ & $0.1676(7)$ & $3.6(12)$ \\
\hline $\mathrm{C}_{2}$ & $0.868(3)$ & $0.7481(25)$ & $0.2168(7)$ & $3.9(13)$ \\
\hline $\mathrm{C} 3$ & $0.666(3)$ & $0.8016(22)$ & $0.1418(8)$ & $3.5(12)$ \\
\hline $\mathrm{C} 4$ & $1.071(3)$ & $0.937(3)$ & $0.1614(8)$ & $4.9(15)$ \\
\hline $\mathrm{C} 5$ & $0.838(3)$ & $0.9471(25)$ & $0.0852(8)$ & $4.7(16)$ \\
\hline C6 & $1.129(3)$ & $0.930(3)$ & $0.0599(9)$ & $5.7(17)$ \\
\hline $\mathrm{C} 7$ & $1.203(3)$ & $0.749(3)$ & $0.1197(8)$ & $4.5(14)$ \\
\hline $\mathrm{C} 8$ & $0.649(4)$ & $0.75](3)$ & $0.0438(8)$ & $6.1(17)$ \\
\hline $\mathrm{CP}$ & $0.906(3)$ & $0.7198(25)$ & $-0.0072(8)$ & $4.8(15)$ \\
\hline $\mathrm{C} 10$ & $0.741(3)$ & $0.537(3)$ & $0.0321(8)$ & $5.0(15)$ \\
\hline $\mathrm{C} 11$ & $1.029(4)$ & $0.578(3)$ & $0.0660(9)$ & $6.1(18)$ \\
\hline $\mathrm{C} 12$ & $0.635(3)$ & $0.294(3)$ & $0.2298(8)$ & $4.9(15)$ \\
\hline $\mathrm{Cl} 3$ & $0.674(3)$ & $0.1071(24)$ & $0.1947(8)$ & $4.1(14)$ \\
\hline $\mathrm{Cl} 4$ & $0.823(3)$ & $0.2168(21)$ & $0.1471(6)$ & $2.9(12)$ \\
\hline $\mathrm{Cl} 5$ & $0.755(3)$ & $0.4196(21)$ & $0.1617(7)$ & $2.7(11)$ \\
\hline $\mathrm{Cl} 6$ & $0.573(3)$ & $0.5590(23)$ & $0.2106(8)$ & $3.8(13)$ \\
\hline $\mathrm{C} 17$ & $0.346(3)$ & $0.199(3)$ & $0.1453(9)$ & $6.2(18)$ \\
\hline $\mathrm{C} 18$ & $0.428(4)$ & $0.152(3)$ & $0.1107(11)$ & $6.7(18)$ \\
\hline $\mathrm{C} 19$ & $0.490(3)$ & $0.235(3)$ & $0.0826(8)$ & $5.3(16)$ \\
\hline $\mathrm{C} 20$ & $0.440(3)$ & $0.331(3)$ & $0.0998(9)$ & $5.3(16)$ \\
\hline $\mathrm{C} 21$ & $0.356(3)$ & $0.318(3)$ & $0.1381(9)$ & $5.3(16)$ \\
\hline 01 & $1.1202(1)$ & $0.5449(20)$ & $0.1801(6)$ & $6.3(11)$ \\
\hline $\mathrm{O} 2$ & $0.890(3)$ & $0.7773(23)$ & $0.2554(5)$ & $8.2(15)$ \\
\hline $\mathrm{O} 3$ & $0.5635(21)$ & $0.8685(17)$ & $0.1381(6)$ & $5.0(11)$ \\
\hline O4 & $1.102(3)$ & $0.9902(20)$ & $0.1953(6)$ & $7.1(12)$ \\
\hline O5 & $0.7273(24)$ & $1.0013(19)$ & $0.0702(6)$ & $6.5(12)$ \\
\hline 06 & $1.2010(23)$ & $0.9780(24)$ & $0.0325(6)$ & $8.2(15)$ \\
\hline 07 & $1.3152(21)$ & $0.6978(19)$ & $0.1285(8)$ & $8.1(13)$ \\
\hline O8 & $0.5290(25)$ & $0.7944(23)$ & $0.0367(7)$ & $8.8(14)$ \\
\hline 09 & $0.958(3)$ & $0.754(3)$ & $-0.0404(7)$ & $12.3(20)$ \\
\hline 010 & $0.669(4)$ & $0.456(3)$ & $0.0207(8)$ & $12.2(21)$ \\
\hline 011 & $1.140(3)$ & $0.5154(23)$ & $0.0739(7)$ & $9.6(15)$ \\
\hline 012 & $0.644(3)$ & $0.3136(19)$ & $0.2699(6)$ & $7.5(14)$ \\
\hline 013 & $0.7031(23)$ & $0.0251(17)$ & $0.2153(5)$ & $5.4(11)$ \\
\hline $\mathrm{O} 14$ & $0.9547(20)$ & $0.1958(19)$ & $0.1357(6)$ & $5.8(11)$ \\
\hline
\end{tabular}

" $B(\mathrm{eq})$ is the mean of the principal axes of the thermal ellipsoid. 
Table III. Atomic Coordinates and Equivalent Isotropic Displacement Coefficients for 3

\begin{tabular}{|c|c|c|c|c|}
\hline & $x$ & $y$ & $z$ & $B(\mathrm{eq})^{a}$ \\
\hline Osl & $0.23176(4)$ & $0.99308(9)$ & $0.21657(6)$ & $1.95(3)$ \\
\hline Os2 & $0.31884(5)$ & $0.90279(10)$ & $0.38760(6)$ & $2.42(4)$ \\
\hline Os3 & $0.39323(5)$ & $0.95938(10)$ & $0.24765(6)$ & $2.50(4)$ \\
\hline w & $0.10361(5)$ & $1.18007(10)$ & $0.16254(6)$ & $2.37(4)$ \\
\hline S & $0.2334(3)$ & $1.2563(6)$ & $0.2319(4)$ & $2.47(24)$ \\
\hline $\mathrm{Cl}$ & $0.1975(10)$ & $0.8062(22)$ & $0.2032(14)$ & $2.4(9)$ \\
\hline $\mathrm{C} 2$ & $0.2506(9)$ & $0.9877(22)$ & $0.1048(17)$ & $3.4(11)$ \\
\hline $\mathrm{C} 3$ & $0.3215(11)$ & $1.109(3)$ & $0.4183(13)$ & $3.1(10)$ \\
\hline $\mathrm{C} 4$ & $0.2393(12)$ & $0.8751(25)$ & $0.4440(17)$ & $3.8(12)$ \\
\hline $\mathrm{C} 5$ & $0.3167(12)$ & $0.692(3)$ & $0.3529(17)$ & $4.4(12)$ \\
\hline $\mathrm{C} 6$ & $0.4074(13)$ & $0.861(3)$ & $0.4785(18)$ & $4.5(13)$ \\
\hline C7 & $0.3657(12)$ & $0.763(3)$ & $0.2006(17)$ & $4.2(13)$ \\
\hline $\mathrm{C} 8$ & $0.4879(11)$ & $0.9091(25)$ & $0.3186(17)$ & $3.8(12)$ \\
\hline $\mathrm{C} 9$ & $0.4243(13)$ & $1.003(3)$ & $0.1415(16)$ & $4.3(12)$ \\
\hline $\mathrm{C} 10$ & $0.4069(11)$ & $1.1585(23)$ & $0.2918(15)$ & $3.0(10)$ \\
\hline $\mathrm{C} 11$ & $0.0661(12)$ & $0.984(3)$ & $0.1120(14)$ & $3.3(11)$ \\
\hline $\mathrm{Cl} 2$ & $0.1273(9)$ & $1.1917(22)$ & $0.0460(15)$ & $2.7(10)$ \\
\hline $\mathrm{C} 13$ & $0.2742(11)$ & $1.3472(25)$ & $0.1511(16)$ & $3.8(11)$ \\
\hline $\mathrm{Cl} 4$ & $0.1476(11)$ & $1.0472(25)$ & $0.2831(14)$ & $3.0(10)$ \\
\hline $\mathrm{C} 15$ & $0.0234(14)$ & $1.268(4)$ & $0.2500(19)$ & $7.1(18)$ \\
\hline $\mathrm{C} 16$ & $0.0696(14)$ & $1.378(3)$ & $0.2381(20)$ & $6.0(15)$ \\
\hline $\mathrm{C} 17$ & $0.0540(11)$ & $1.4076(24)$ & $0.1459(18)$ & $3.9(13)$ \\
\hline C18 & $-0.0022(12)$ & $1.310(3)$ & $0.1041(17)$ & $5.2(13)$ \\
\hline $\mathrm{C} 19$ & $-0.0196(13)$ & $1.225(3)$ & $0.1705(20)$ & $5.8(16)$ \\
\hline $\mathrm{OI}$ & $0.1708(8)$ & $0.6890(16)$ & $0.1972(12)$ & $4.4(9)$ \\
\hline $\mathrm{O} 2$ & $0.2579(9)$ & $0.9715(18)$ & $0.0296(11)$ & $4.8(9)$ \\
\hline $\mathrm{O} 3$ & $0.3231(10)$ & $1.2232(18)$ & $0.4454(11)$ & $5.2(10)$ \\
\hline O4 & $0.1911(9)$ & $0.8580(24)$ & $0.4831(12)$ & $6.8(12)$ \\
\hline O5 & $0.3136(11)$ & $0.5771(17)$ & $0.3384(14)$ & $6.9(13)$ \\
\hline 06 & $0.4585(8)$ & $0.8357(19)$ & $0.5354(12)$ & $5.3(9)$ \\
\hline 07 & $0.3540(11)$ & $0.6554(18)$ & $0.1666(13)$ & $6.5(12)$ \\
\hline 08 & $0.5440(8)$ & $0.8699(19)$ & $0.3668(13)$ & $5.5(10)$ \\
\hline O9 & $0.4464(11)$ & $1.0332(21)$ & $0.0795(13)$ & $6.7(12)$ \\
\hline 010 & $0.4182(8)$ & $1.2774(16)$ & $0.3158(11)$ & $3.9(8)$ \\
\hline 011 & $0.0416(8)$ & $0.8801(17)$ & $0.0805(11)$ & $4.8(8)$ \\
\hline 012 & $0.1367(9)$ & $1.2087(22)$ & $-0.0269(11)$ & $5.7(10)$ \\
\hline
\end{tabular}

" $B($ eq $)$ is the mean of the principal axes of the thermal ellipsoid. 
Table IV. Atomic Coordinates and Equivalent Isotropic Displacement Coefficients for 4

\begin{tabular}{|c|c|c|c|c|}
\hline & $x$ & $y$ & $=$ & $B(\mathrm{eq})^{a}$ \\
\hline Os 1 & $0.31137(4)$ & $0.16672(8)$ & $0.00291(4)$ & $2.75(3)$ \\
\hline Os2 & $0.36242(4)$ & $0.36370(8)$ & $0.14283(4)$ & $3.11(3)$ \\
\hline Os 3 & $0.23940(4)$ & $0.46564(8)$ & $0.01739(4)$ & $2.79(3)$ \\
\hline W & $0.14664(4)$ & $0.20679(8)$ & $0.07186(4)$ & $2.73(3)$ \\
\hline S & $0.1095(3)$ & $0.3845(6)$ & $-0.04201(24)$ & $3.97(20)$ \\
\hline $\mathrm{Cl}$ & $0.2809(12)$ & $0.0757(21)$ & $-0.1075(10)$ & $4.5(9)$ \\
\hline $\mathrm{C} 2$ & $0.3287(10)$ & $-0.0196(21)$ & $0.0642(9)$ & $3.9(8)$ \\
\hline $\mathrm{C} 3$ & $0.4211(11)$ & $0.1914(21)$ & $-0.0252(11)$ & $4.6(9)$ \\
\hline $\mathrm{C} 4$ & $0.3960(10)$ & $0.2157(23)$ & $0.2255(9)$ & $4.3(9)$ \\
\hline $\mathrm{C} 5$ & $0.4713(11)$ & $0.4258(22)$ & $0.1150(9)$ & $4.5(9)$ \\
\hline $\mathrm{C} 6$ & $0.3495(11)$ & $0.5154(22)$ & $0.2266(9)$ & $4.3(8)$ \\
\hline $\mathrm{C} 7$ & $0.2636(10)$ & $0.6130(20)$ & $-0.0657(9)$ & $3.5(7)$ \\
\hline $\mathrm{C} 8$ & $0.1881(2)$ & $0.6090(19)$ & $0.0872(9)$ & $4.3(9)$ \\
\hline $\mathrm{C} 9$ & $0.1531(9)$ & $0.0198(20)$ & $0.0016(9)$ & $3.3(7)$ \\
\hline $\mathrm{ClO}$ & $0.2559(10)$ & $0.2782(19)$ & $0.1103(8)$ & $3.2(7)$ \\
\hline $\mathrm{Cll}$ & $0.1163(13)$ & $0.3210(24)$ & $-0.1516(10)$ & $5.7(11)$ \\
\hline $\mathrm{C} 12$ & $0.0143(10)$ & $0.2441(22)$ & $0.1208(10)$ & $4.4(9)$ \\
\hline $\mathrm{C} 13$ & $0.0693(10)$ & $0.321(3)$ & $0.1752(11)$ & $5.6(10)$ \\
\hline $\mathrm{C} 14$ & $0.1224(10)$ & $0.214(3)$ & $0.2166(9)$ & $5.7(11)$ \\
\hline $\mathrm{C} 15$ & $0.1013(12)$ & $0.0723(24)$ & $0.1874(10)$ & $5.4(10)$ \\
\hline $\mathrm{Cl} 6$ & $0.0339(10)$ & $0.0919(23)$ & $0.1282(10)$ & $4.5(9)$ \\
\hline $\mathrm{Ol}$ & $0.2626(8)$ & $0.0304(14)$ & $-0.1745(6)$ & $5.1(7)$ \\
\hline $\mathrm{O} 2$ & $0.3386(8)$ & $-0.1343(16)$ & $0.1028(8)$ & $6.1(8)$ \\
\hline $\mathrm{O} 3$ & $0.4861(8)$ & $0.1944(21)$ & $-0.0438(9)$ & $8.3(9)$ \\
\hline $\mathrm{O} 4$ & $0.4143(8)$ & $0.1214(16)$ & $0.2741(7)$ & $6.0(7)$ \\
\hline 05 & $0.5349(8)$ & $0.4552(18)$ & $0.0971(8)$ & $6.7(8)$ \\
\hline O6 & $0.3363(10)$ & $0.6118(18)$ & $0.2762(7)$ & $7.3(9)$ \\
\hline 07 & $0.2804(9)$ & $0.7034(15)$ & $-0.1175(7)$ & $5.8(7)$ \\
\hline 08 & $0.1585(9)$ & $0.6916(15)$ & $0.1346(7)$ & $6.4(8)$ \\
\hline 09 & $0.1469(7)$ & $-1.1000(17)$ & $-0.0321(8)$ & $5.8(7)$ \\
\hline
\end{tabular}

" $B(\mathrm{eq})$ is the mean of the principal axes of the thermal ellipsoid.

$R=0.048$ and $R_{W}=0.044$, for 2582 reflections with $I>2 \sigma(I)$. A weighting scheme based on counting statistics was used with the weight modifier $k$ in $k F_{0}^{2}$ being 0.0001 . The residual electron density on the difference Fourier map is $-1.48 / 2.47 e / \AA^{3}$.

Lattice parameters for complex 3 were determined from 25 randomly selected high angle reflections with $2 \theta$ angles in the range $17.6-22.8^{\circ}$. The minimum and maximum transmission factors were 0.39 and 1.00 , respectively. Full matrix least-squares refinement with 46 atoms and 326 parameters gave $R=0.047$ and $R_{H}=0.045$ for 3187 reflections with $I>2 \sigma(I)$. A weighting scheme based on counting statistics was used with 
Table V. Atomic Coordinates and Equivalent Isotropic Displacement Coefficients for 5

\begin{tabular}{|c|c|c|c|c|}
\hline & $x$ & $y$ & $=$ & $B(\mathrm{eq})^{a}$ \\
\hline Os1 & $0.13834(7)$ & $3 / 4$ & $0.19675(7)$ & $1.88(3)$ \\
\hline Os2 & $0.25808(4)$ & $0.62273(4)$ & $0.06911(4)$ & $1.630(22)$ \\
\hline W & $0.19363(7)$ & $3 / 4$ & $-0.12859(7)$ & $1.71(3)$ \\
\hline S & $0.3705(4)$ & $3 / 4$ & $0.1085(4)$ & $1.84(22)$ \\
\hline $\mathrm{Cl}$ & $0.1923(24)$ & $3 / 4$ & $0.3544(18)$ & $4.6(16)$ \\
\hline $\mathrm{C} 2$ & $0.0523(11)$ & $0.6491(11)$ & $0.2234(12)$ & $2.6(7)$ \\
\hline $\mathrm{C} 3$ & $0.2729(11)$ & $0.5773(10)$ & $0.2185(12)$ & $2.2(6)$ \\
\hline $\mathrm{C} 4$ & $0.1597(12)$ & $0.5339(10)$ & $0.0404(11)$ & $2.3(7)$ \\
\hline $\mathrm{C} 5$ & $0.3469(13)$ & $0.5359(10)$ & $0.0020(12)$ & $2.9(8)$ \\
\hline C6 & $0.2862(12)$ & $0.6596(11)$ & $-0.1798(12)$ & $2.6(7)$ \\
\hline $\mathrm{C7}$ & $0.1716(19)$ & $3 / 4$ & $0.0379(16)$ & $3.0(12)$ \\
\hline $\mathrm{C} 8$ & $0.4597(18)$ & $3 / 4$ & $0.0032(17)$ & $2.8(11)$ \\
\hline $\mathrm{C} 9$ & $0.0317(23)$ & $3 / 4$ & $-0.1381(22)$ & $7.4(21)$ \\
\hline $\mathrm{ClO}$ & $0.0637(18)$ & $0.8306(13)$ & $-0.1952(17)$ & $7.0(13)$ \\
\hline $\mathrm{Cll}$ & $0.1110(16)$ & $0.8010(13)$ & $-0.2852(15)$ & $6.0(11)$ \\
\hline OI & $0.2253(15)$ & $3 / 4$ & $0.4367(12)$ & $6.3(13)$ \\
\hline $\mathrm{O} 2$ & $0.0019(10)$ & $0.5852(8)$ & $0.2378(10)$ & $4.5(6)$ \\
\hline $\mathrm{O} 3$ & $0.2920(9)$ & $0.5522(8)$ & $0.3084(8)$ & $3.7(6)$ \\
\hline 04 & $0.1017(9)$ & $0.4802(8)$ & $0.0251(9)$ & $3.7(6)$ \\
\hline 05 & $0.3983(10)$ & $0.4821(9)$ & $-0.0320(10)$ & $5.0(7)$ \\
\hline O6 & $0.3430(9)$ & $0.6092(8)$ & $-0.2273(9)$ & $4.2(6)$ \\
\hline
\end{tabular}

" $B(e q)$ is the mean of the principal axes of the thermal ellipsoid.

the weight modifier $k$ in $k F_{o}^{2}$ being 0.00002 . The residual electron density is $-3.13 / 3.28 e / \AA^{3}$.

Lattice parameters of $\mathbf{4}$ were determined from 25 randomly selected high angle reflections with $2 \theta$ angles in the range $11.0-24.8^{\circ}$. The minimum and maximum transmission factors were 0.25 and 1.00 , respectively. Refinement with 38 atoms and 272 parameters gave $R=0.036$ and $R_{W}=0.034$,

Table VI. Selected Bond Distance $(A)$ and Bond Angles $\left({ }^{\circ}\right)$ for 2 (esd in Parentheses)

\begin{tabular}{lclc}
\hline Os(1)-Os(2) & $2.847(2)$ & Os(1)-Os(3) & $2.894(1)$ \\
Os(2)-Os(3) & $2.885(2)$ & Os(1)-S & $2.399(7)$ \\
S-C(15) & $1.77(3)$ & S-C(16) & $1.76(2)$ \\
W-C(15) & $2.33(2)$ & Os-CO(mean) & $1.90(3)$ \\
W-CO(mean) & $1.95(3)$ & & \\
Os(1)-Os(2)-Os(3) & $60.65(4)$ & Os(2)-Os(1)-Os(3) & $60.33(4)$ \\
Os(1)-Os(3)-Os(2) & $59.02(4)$ & Os(1)-S-C15) & $109.7(8)$ \\
Os(1)-S-C(16) & $107.6(9)$ & C $(15)-\mathrm{S}-\mathrm{C}(16)$ & $102.5(11)$ \\
W-C(15)-S & $120.2(11)$ & Mean Os-CO & $176(2)$ \\
Mean W-CO & $177(2)$ & & \\
\hline
\end{tabular}


Table VII. Selected Bond Distances $(A)$ and Bond Angles $\left({ }^{\circ}\right)$ for 3 (esd in Parentheses)

\begin{tabular}{lclc}
\hline Os(1)-Os(2) & $2.855(1)$ & Os(1)-Os(3) & $2.875(1)$ \\
Os(2)-Os(3) & $2.840(1)$ & Os(1)-W & $2.863(1)$ \\
Os(1)-S & $2.447(6)$ & W-S & $2.452(5)$ \\
Os(1)-C(14) & $2.09(2)$ & W-C(14) & $2.21(2)$ \\
S-C(13) & $1.80(2)$ & Os-CO(mean) & $1.91(3)$ \\
W-CO(mean) & $1.99(3)$ & & \\
Os(1)-Os(2)-Os(3) & $60.65(3)$ & Os(2)-Os(1)-Os(3) & $59.41(4)$ \\
Os(1)-Os(3)-Os(2) & $59.94(3)$ & W-Os(1)-Os(2) & $132.97(4)$ \\
W-Os(1)-Os(3) & $47.35(4)$ & W-C(14)-Os(1) & $83.5(7)$ \\
W-S-Os(1) & $71.5(1)$ & Os(1)-S-C(13) & $113.4(8)$ \\
W-S-C(13) & $110.1(7)$ & Mean Os-CO & $175(2)$ \\
Mean W-CO & $174(2)$ & & \\
\hline
\end{tabular}

Table VIII. Selected Bond Distances $(\hat{A})$ and Bond Angles $\left({ }^{\circ}\right)$ for 4 (esd in Parentheses)

\begin{tabular}{lcll}
\hline $\mathrm{Os}(1)-\mathrm{Os}(2)$ & $2.847(1)$ & $\mathrm{Os}(1)-\mathrm{Os}(3)$ & $2.873(1)$ \\
$\mathrm{Os}(2)-\mathrm{Os}(3)$ & $2.870(1)$ & $\mathrm{Os}(1)-\mathrm{W}$ & $2.998(1)$ \\
$\mathrm{Os}(3)-\mathrm{W}$ & $2.875(1)$ & $\mathrm{Os}(1)-\mathrm{C}(10)$ & $2.17(1)$ \\
$\mathrm{Os}(2)-\mathrm{C}(10)$ & $1.95(2)$ & $\mathrm{Os}(3)-\mathrm{C}(10)$ & $2.18(2)$ \\
$\mathrm{W}-\mathrm{C}(10)$ & $1.97(2)$ & $\mathrm{Os}(3)-\mathrm{S}$ & $1.395(5)$ \\
$\mathrm{W}-\mathrm{S}$ & $2.394(4)$ & $\mathrm{Os}(1)-\mathrm{C}(1)$ & $1.92(2)$ \\
$\mathrm{Os}(1)-\mathrm{C}(2)$ & $1.89(2)$ & $\mathrm{Os}(1)-\mathrm{C}(3)$ & $1.90(2)$ \\
$\mathrm{Os}(2)-\mathrm{C}(4)$ & $1.87(2)$ & $\mathrm{Os}(2)-\mathrm{C}(5)$ & $1.95(2)$ \\
$\mathrm{Os}(2)-\mathrm{C}(6)$ & $1.87(2)$ & $\mathrm{Os}(3)-\mathrm{C}(7)$ & $1.87(2)$ \\
$\mathrm{Os}(8)-\mathrm{C}(8)$ & $1.88(2)$ & $\mathrm{W}-\mathrm{C}(9)$ & $1.96(2)$ \\
$\mathrm{S}-\mathrm{C}(11)$ & $1.79(2)$ & & \\
Os(2)-C(10)-W & $175.4(9)$ & $\mathrm{Os}(1)-\mathrm{C}(10)-\mathrm{Os}(3)$ & $82.8(4)$ \\
Os(3)-S-W & $73.8(1)$ & & \\
\hline
\end{tabular}

Table IX. Selected Bond Distances (A) and Bond Angles $\left({ }^{\circ}\right.$ ) for 5 (esd in Parentheses)

\begin{tabular}{lcll} 
Os(1)-Os(2) & $2.886(1)$ & Os(2)-Os(2) & $3.490(2)$ \\
Os(2)-W & $3.054(1)$ & Os(1)-C(7) & $1.93(2)$ \\
Os(2)-C(7) & $2.18(1)$ & W-C(7) & $1.98(2)$ \\
Os(2)-S & $2.435(4)$ & $\mathrm{S}-\mathrm{C}(8)$ & $1.79(2)$ \\
Os(1)-C(1) & $2.01(3)$ & Os(1)-C(2) & $1.89(2)$ \\
Os(2)-C(3) & $1.88(2)$ & Os(2)-C(4) & $1.91(2)$ \\
Os(2)-C(5) & $1.93(2)$ & W-C(6) & $1.93(2)$ \\
Os(1)-C(7)-W & $175(2)$ & Os(2)-C(7)-Os(2) & $106(1)$ \\
Os(2)-S-Os(2) & $91.5(2)$ & & \\
\hline
\end{tabular}


for 2780 reflections with $I>2 \sigma(I)$. A weighting scheme based on counting statistics was used with the weight modifier $k$ in $k F_{o}^{2}$ being 0.00005 . The residual electron density is $-1.90 / 1.56 \mathrm{e} / \AA^{3}$.

For complex 5, the lattice parameters were determined from 25 randomly selected high angle reflections with $2 \theta$ angles in the range $18.5-30.0^{\circ}$. The minimum and maximum transmission factors were 0.15 and 1.00 , respectively. Refinement with 26 atoms and 167 parameters gave $R=0.038$ and $R_{W}=0.037$ for 1826 reflections with $I>2 \sigma(I)$. A weighting scheme based on counting statistics was used with the weight modifier $k$ in $k F_{o}^{2}$ being 0.00001 . The residual electron density is $-2.81 / 1.96 e / \AA^{3}$.

\section{RESULTS AND DISCUSSION}

Condensation of $\mathrm{Os}_{3}(\mathrm{CO})_{10}(\mathrm{NCMe})_{2}$ and $\mathrm{CpW}(\mathrm{CO})_{3}\left(\mathrm{CH}_{2} \mathrm{SMe}\right)$. Treatment of the triosmium acetonitrile complex $\mathrm{Os}_{3}(\mathrm{O})_{10}(\mathrm{NCMe})_{2}$ with 1.2 equivalent of the tungsten sulfido complex $\mathrm{CpW}(\mathrm{CO})_{3}\left(\mathrm{CH}_{2} \mathrm{SMe}\right)$ in refluxing THF solution produced the thiolate cluster $\mathrm{Os}_{3}(\mathrm{CO})_{10}(\mu-\mathrm{H})$ $\left(\mu\right.$-SMe) $(1)$ in $41 \%$ yield and two additional $\mathrm{WOs}_{3}$ heterometallic clusters $\mathrm{Os}_{3}(\mathrm{CO})_{11}\left[\mathrm{~S}(\mathrm{Me}) \mathrm{CH}_{2} \mathrm{~W}(\mathrm{CO})_{3} \mathrm{Cp}\right]$ (2) and $\mathrm{CpWO}_{3}(\mathrm{CO})_{12}\left(\mu-\mathrm{CH}_{2}\right)$ $(\mu$-SMe) (3) in $19 \%$ and $8 \%$ yield, respectively (Scheme I). The reactivity of the sulfido reagent $\mathrm{CpW}(\mathrm{CO})_{3}\left(\mathrm{CH}_{2} \mathrm{SMe}\right)$ is almost identical to that of the related decarbonylated species $\mathrm{CpW}(\mathrm{CO})_{2}\left(\eta^{2}-\mathrm{CH}_{2} \mathrm{SMe}\right)$, which contains two $\mathrm{CO}$ ligands and a $\mathrm{C}$ and S-bound $\mathrm{CH}_{2}$ SMe group. Attempts to carry out the reaction in toluene at reflux failed to give any heterometallic complexes $\mathbf{2}$ and 3 , but produced homometallic $\mathbf{1}$ as the only isolable product, suggesting that this condensation reaction is sensitive to both the solvent and the temperature employed. These three cluster compounds were separated by thin-layer chromatography on silica gel and were fully characterized by microanalysis, spectroscopic, and structural methods.

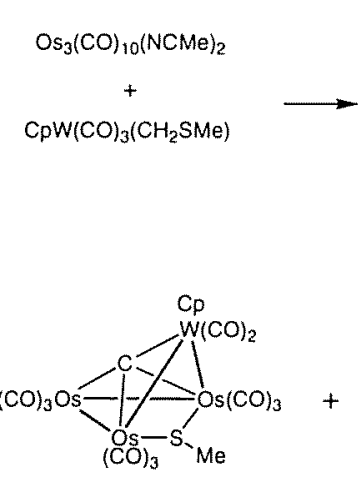

(5)

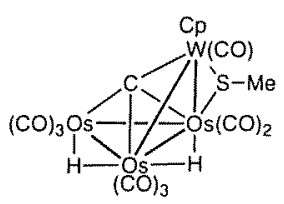

(4)

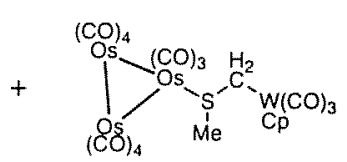

(2)

(1)

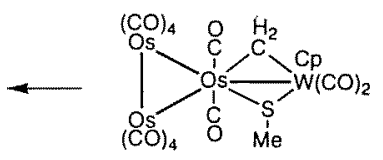

(3)

Scheme 1 
Compound 1 is related to the derivatives of the type $\mathrm{Os}_{3}(\mathrm{CO})_{10}$ $(\mu-\mathrm{H})(\mu-\mathrm{S} R)$ and $\mathrm{Ru}_{3}(\mathrm{CO})_{10}(\mu-\mathrm{H})(\mu-\mathrm{S} R), \quad R=\mathrm{Et}$ and $\mathrm{Ph}$, which have been well-characterized by Lewis and Johnson, and by Adams and co-workers independently [9]. The key spectral feature of 1 involves the observation of a methyl signal at $\delta 2.43$ and a hydride signal at $\delta-17.16$ in the ${ }^{1} \mathrm{H}$ NMR spectrum. The ${ }^{13} \mathrm{C}$ NMR spectrum measured at room temperature showed six $\mathrm{CO}$ signals at $\delta 180.8,180.2,176.3,173.7,170.7,169.2$ in a ratio $1: 1: 2: 2: 2: 2$. This six-line pattern reveals the presence of $C_{s}$ symmetry, which is typical for this type of cluster compounds with formula $\mathrm{Os}_{3}(\mathrm{CO})_{10}(\mu-\mathrm{H})(\mu-X), \quad X=$ halides, alkoxides and thiolate functional groups [10].

Characterization of $\mathrm{Os}_{3}(\mathrm{CO})_{11}\left[\mathrm{~S}(\mathrm{Me}) \mathrm{CH}_{2} \mathrm{~W}(\mathrm{CO})_{3} \mathrm{Cp}\right]$ (2). Complex 2 can be considered as a derivative of $\mathrm{Os}_{3}(\mathrm{CO})_{11}(\mathrm{~L})$ and the unique ligand is a sulfur-containing fragment. The molecular structure is depicted in Fig. 1 and the selected data of bond lengths and angles is presented in Table VI. The molecule has an isosceles triangular geometry, in which the sulfur atom of ligand $\mathrm{CpW}(\mathrm{CO})_{3}\left(\mathrm{CH}_{2} \mathrm{SMe}\right)$ is coordinated to the $\mathrm{Os}_{3}$ metal triangle through trivalent sulfur atom at an equatorial site. The tungsten center is considered to adopt a characteristic four-leg piano stool structure. The Os(1)-Os(2) distance of $2.847(2) \AA$ is slightly longer than the other two Os-Os distances within this molecule $(\mathrm{Os}(1)-\mathrm{Os}(3)=$ $2.894(1) \AA$ and $\operatorname{Os}(2)-\mathrm{Os}(3)=2.885(1) \AA$ ), which are comparable to the average Os-Os distance $2.877 \AA$ observed in the parent cluster $\mathrm{Os}_{3}(\mathrm{CO})_{12}$ [11]. The shortening of the Os(1)-Os(2) distance is a result of $\pi$-bond competition because the sulfur atom of the sulfido ligand tends to form a much weaker metal-to-ligand back $\pi$-bonding with respect to the $\mathrm{CO}$ ligand. Therefore, the Os(1) atom would interact much stronger with the

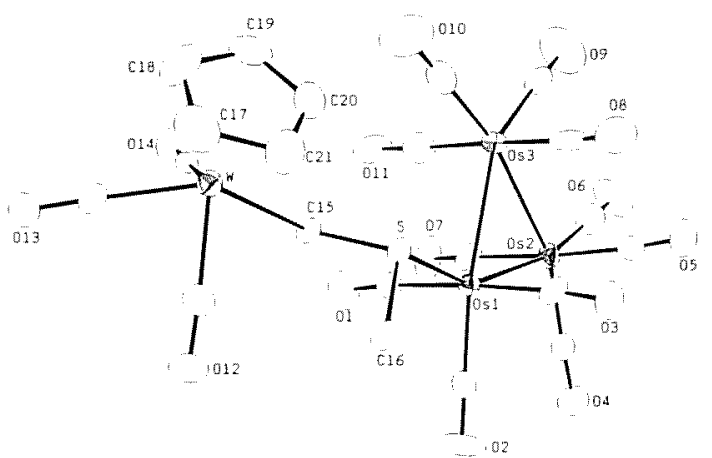

Fig. 1. Molecular structure of 2 and the atomic numbering scheme. 
Os(2) atom, which is located at the trans-position, and this results in the short Os-Os bond. Similar variation in Os-Os and $\mathrm{Ru}-\mathrm{Ru}$ distances has been noted for the phosphine derivatives $\mathrm{Os}_{3}(\mathrm{CO})_{11}\left[\mathrm{P}(\mathrm{OMe})_{3}\right]$ [2] and $\mathrm{Ru}_{3}(\mathrm{CO})_{11}\left(\mathrm{PPh}_{3}\right)[3]$.

In accordance with the result of X-ray diffraction study, the ${ }^{13} \mathrm{C}$ NMR spectrum of a ${ }^{13} \mathrm{CO}$ enriched sample at $230 \mathrm{~K}$ displays two $\mathrm{W}-\mathrm{CO}$ resonances at $\delta 225.6$ and 217.3 in the ratio $1: 2$, and a set of nine Os-CO signals at $\delta 194.7\left(d, J_{C-C}=36.5 \mathrm{~Hz}\right), 185.5\left(d, J_{C-C}=36.5 \mathrm{~Hz}\right), 185.5(2 \mathrm{C})$, $183.5(2 \mathrm{C}), 177.0,176.3,171.8,171.3,170.8$. For the first two Os-CO resonances, the observed ${ }^{2} J_{C-C}$ coupling falls in the range expected for the diaxial trans-CO ligands, thus they are assigned to the axial $\mathrm{CO}$ ligands of the Os(1) atom. The Os-CO signals at $\delta 185.5$ and 183.5 , which possess an intensity corresponding to two $\mathrm{CO}$ ligands, are assigned to the axial $\mathrm{CO}$ ligands of the atoms $\mathrm{Os}(2)$ and $\mathrm{Os}(3)$, as the molecules may contain a time averaged plane of mirror symmetry in solution which coincides with the osmium triangle. Upon raising the temperature to $294 \mathrm{~K}$, all Os-CO signals broaden and merge into the baseline simultaneously. This behavior is in consistent with the coexistence of rapid intra and intermetallic $\mathrm{CO}$ exchange on the ligand sphere of osmium triangle. No attempt was made to further elucidate the mechanistic details of the $\mathrm{CO}$ exchange.

Characterization of $\mathrm{CpWO}_{3}(\mathrm{CO})_{12}\left(\mu-\mathrm{CH}_{2}\right)(\mu-\mathrm{SMe})$ (3). The ${ }^{1} \mathrm{H}$ NMR spectrum of 3 exhibits $\mathrm{Cp}$ and methyl resonances at $\delta 5.47$ and 2.19 in addition to two methylene hydrogen signals at $\delta 3.84$ and 3.41 with ${ }^{2} J_{H-H}=4.0 \mathrm{~Hz}$. The ${ }^{13} \mathrm{C}$ NMR spectrum clearly shows the presence of seven $\mathrm{CO}$ signals. Two downfield resonance signals of equal intensity at $\delta 222.0$ and 215.2 are due to the $\mathrm{W}-\mathrm{CO}$ ligands. Five other highfield $\mathrm{CO}$ signals at $\delta 187.2,185.1,183.8(4 \mathrm{C}), 174.1(2 \mathrm{C})$ and $167.8(2 \mathrm{C}, \mathrm{br})$ are assigned to the Os-CO ligands. Based on these spectral information, a formula $\mathrm{CpWOs}_{3}(\mathrm{CO})_{12}\left(\mu-\mathrm{CH}_{2}\right)(\mu$-SMe $)$, which involves a $\mathrm{CpWOs}_{3}(\mathrm{CO})_{12}$ core, one bridging methylene and methylthiolato group, is proposed for this complex.

Complex 2 was further characterized by single crystal X-ray diffraction. As indicated in Fig. 2, this molecule adopts a spiked-triangular core arrangement, in which the atoms $\mathrm{Os}(2)$ and $\mathrm{Os}(3)$ are each associated with four terminal CO ligand. The Os(1) atom, which is located at the pivotal position, carries two $\mathrm{CO}$ ligands, and the $\mathrm{W}$ atom bears a cyclopentadienyl and two terminal $\mathrm{CO}$ ligands. All metal-metal bonds fall in a narrow range 2.840-2.875 $\AA$ (Table VII), showing the metal-metal single bond interactions. The methylene and methylthiolato ligands are both coordinated to 


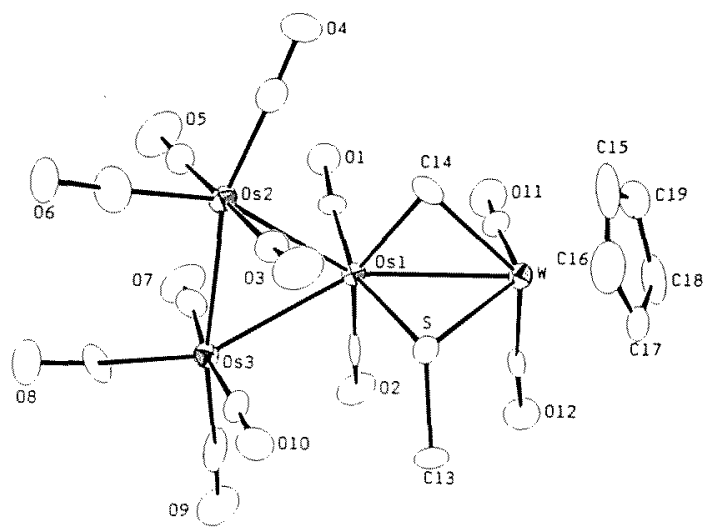

Fig. 2. Molecular structure of 3 and the atomic numbering scheme.

the metal atoms, $\mathrm{Os}(1)$ and $\mathrm{W}$. The methylthiolate ligands bridges symmetrically with typical distances $\mathrm{Os}(1)-\mathrm{S}=2.447(6) \AA$ and $\mathrm{W}-\mathrm{S}=$ $2.452(5) \AA$. The methylene ligand is asymmetrical with $\mathrm{Os}(1)-\mathrm{C}(14)$ bond $(2.09(2) \AA)$ and is substantially shorter than that of $\mathrm{W}-\mathrm{C}(14)$ bond $(2.21(2) \AA)$. The $\mathrm{M}-\mathrm{C}$ bond distances are in the range observed for the M-C (alkylidene) distances of $\mathrm{CpWO}_{3}(\mu-\mathrm{O})(\mu-\mathrm{H})(\mu-\mathrm{CHTol})$ ( $\mathrm{W}-\mathrm{C}=2.08 \AA$ and $\mathrm{Os}-\mathrm{C}=2.24 \AA$ ) [4] and the Os-C distances of $\left.\mathrm{Os}_{3}(\mathrm{CO})_{11}\left(\mu-\mathrm{CHSiMe}_{3}\right)(2.16-2.19)(2) \AA\right)$ [15], which possess unsymmetrical and symmetrical alkylidene group, respectively.

Generation of Carbido Clusters 4 and 5 . Thermolysis of 3 was conducted in refluxing toluene solution for 1.5 hours. After chromatographic separation, we isolated two carbido clusters $\mathrm{CpWO}_{3}(\mathrm{CO})_{9}\left(\mu_{4}-\mathrm{C}\right)$ $(\mu-\mathrm{H})_{2}(\mu-\mathrm{SMe})(4)$ and $\mathrm{CpWO}_{3}(\mathrm{CO})_{11}\left(\mu_{4}-\mathrm{C}\right)(\mu$-SMe $)(5)$ in moderate yields. The chemistry demonstrated yet another example of methylene to carbide transformation, which is parallel to other observed reactivity of methylene and methylidyne cluster compounds, viz., that $\mathrm{Os}_{3}(\mathrm{CO})_{10}$ $(\mu-\mathrm{H})_{2}\left(\mu-\mathrm{CH}_{2}\right)$ was converted to methylidyne cluster $\mathrm{Os}_{3}(\mathrm{CO})_{9}(\mu-\mathrm{H})_{3}$ $\left(\mu_{3}-\mathrm{CH}\right)$ upon heating, that methylene complex $\mathrm{Cp}_{2} \mathrm{Ru}_{2}(\mathrm{CO})_{2}$ $\left(\mu-\mathrm{CH}_{2}\right)(\mathrm{NCMe})$ reacted with $\mathrm{Pt}\left(\mathrm{C}_{2} \mathrm{H}_{4}\right)_{2}\left(\mathrm{P} R_{3}\right), R=\mathrm{Pr}^{i}$, Cy, to produce tetranuclear carbido cluster $\mathrm{Cp}_{2} \mathrm{Ru}_{2} \mathrm{Pt}_{2}(\mathrm{CO})_{2}\left(\mathrm{PR}_{3}\right)_{2}\left(\mu_{4}-\mathrm{C}\right)(\mu-\mathrm{H})_{2}$ as principle product [16]. Furthermore, the methylidyne cluster $\mathrm{Os}_{3}(\mathrm{O})_{10}$ $(\mu-\mathrm{H})\left(\mu_{3}-\mathrm{CH}\right)$ also was found to condense with $\mathrm{Pt}\left(\mathrm{C}_{2} \mathrm{H}_{4}\right)_{2}\left(\mathrm{PR}_{3}\right)$, affording carbido clusters $\mathrm{PtOs}_{3}(\mathrm{CO})_{10}\left(\mathrm{PR}_{3}\right)\left(\mu_{4}-\mathrm{C}\right)(\mu-\mathrm{H})_{2}$ and $\mathrm{Pt}_{2} \mathrm{Os}_{3}(\mathrm{CO})_{10}\left(\mathrm{PR}_{3}\right)_{2}\left(\mu_{5}-\mathrm{C}\right)(\mu-\mathrm{H})_{2} \quad$ [17]. These interesting results illustrate a route to carbide clusters by metal-promoted double $\mathrm{C}-\mathrm{H}$ bond activation, showing a unique model of stepwise transformation from $\mathrm{CH}_{2}$, to $\mathrm{CH}+\mathrm{H}$ and ultimately to $\mathrm{C}+2 \mathrm{H}[18]$. 
Characterization of 4 . The identification of these two carbido clusters was achieved by using NMR spectroscopies and X-ray diffraction studies. The ${ }^{1} \mathrm{H}$ NMR spectrum of 4 exhibited one Cp signal at $\delta 5.46$, one methyl singlet at $\delta 2.76$ and two hydride resonances at $\delta-17.04$ and -24.14 , indicating the retention of $\mathrm{CpWOs}_{3}$ metal fragment and the conversion of methylene hydrogen atoms into bridging hydride ligands. Also, the ${ }^{13} \mathrm{C}$ NMR data were consistent with the generation of carbido ligand. The ${ }^{13} \mathrm{C} \mathrm{NMR}$ spectrum measured in $\mathrm{CD}_{2} \mathrm{Cl}_{2}$ revealed a signal attributable to the carbido ligand at $\delta 305.4$. This observed chemical shift is similar to the carbido resonance $(\delta 339.3)$ for cluster $\mathrm{Cp}_{2} \mathrm{MoWO}_{3}(\mathrm{CO})_{8}\left(\mu_{4}-\mathrm{C}\right)$ $\left(\mu_{3}-\mathrm{Ph}\right)(\mathrm{CCPh})[19]$.

The structural features of 4 were fully in agreement with its spectral properties. As indicated in Fig. 3, this molecule possesses a butterfly arrangement with the $\mathrm{CpW}(\mathrm{CO})$ fragment located at a wing-tip position and the symmetrical thiolate ligand, $\mu$-SMe, spanned the $\mathrm{W}-\mathrm{Os}(3)$ bond with distances, $O s(3)-S=2.395(5) \AA, \quad W-S=2.394(4) \AA$ and $\mathrm{W}-\mathrm{Os}(3)=2.875(1) \AA$ (Table VIII). The dihedral angle between the $\mathrm{W}-\mathrm{Os}(1)-\mathrm{Os}(3)$ and $\mathrm{Os}(1)-\mathrm{Os}(2)-\mathrm{Os}(3)$ planes is $102.27(3)^{\circ}$. The carbide atom $\mathrm{C}(10)$ located between the wing-tip atoms $\mathrm{W}$ and Os(2) with distances $\mathrm{W}-\mathrm{C}(10)=1.97(2) \AA$ and $\mathrm{Os}(2)-\mathrm{C}(10)=1.95(2) \AA$ and angle $\angle \mathrm{W}-\mathrm{C}(10)-\mathrm{Os}(2)=175.4(9)^{\circ}$, and centered over the hinge Os(1)-Os(3) bond with distances $\mathrm{Os}(1)-\mathrm{C}(10)=2.17(1) \AA$ and $\mathrm{Os}(3)-\mathrm{C}(10)=2.18(2) \AA$ and angle $\angle \mathrm{Os}(1)-\mathrm{C}(10)-\mathrm{Os}(3)=82.8(4)^{\circ}$. These parameters resemble that observed for the carbide atom in the related heterometallic $\mathrm{WRu}_{3}$ and homonuclear $\mathrm{Ru}_{4}$ and $\mathrm{Fe}_{4}$ carbido clusters [20], which showed short $\mathrm{M}$ (wingtip)-C(carbide) distances (av. 1.98(2) $\AA$ ), long $\mathrm{M}$ (hinge)$\mathrm{C}$ (carbide) distances (av. $2.12(2)^{\circ} \AA$ ), and a relatively acute dihedral angle. Finally, the hydride ligands have not been located, but it is likely that the hydrides are associated with the adjacent Os(1)-Os(3) and Os(2)-Os(3) edges. The supporting evidences are provided by the elongation of these Os-Os bonds $(2.870-2.871 \AA$ ) with respect to the third Os-Os bond $\left(2.847(3) \AA\right.$ ) and the absence of $J_{W-C}$ coupling for both hydride signals in the ${ }^{1} \mathrm{H}$ NMR spectrum.

Characterization of 5. The structure of 5 was also examined by single crystal X-ray analysis. This molecule crystallizes in an orthorhombic space group P nma with a crystallographic imposed plane of mirror symmetry. As indicated in Fig. 4, the cluster core composed of a cyclic arrangement of one $\mathrm{W}$ and three Os atoms, which are all bonded to the carbido atom with distances 2.18(1) $\AA-1.93(1) \AA$ (Table IX). In addition, the $W$ atom is coordinated to two $\mathrm{CO}$ and one $\mathrm{Cp}$ ligand, while each Os atoms are coordinated by three orthogonal $\mathrm{CO}$ ligands. The bridging thiolate ligand 


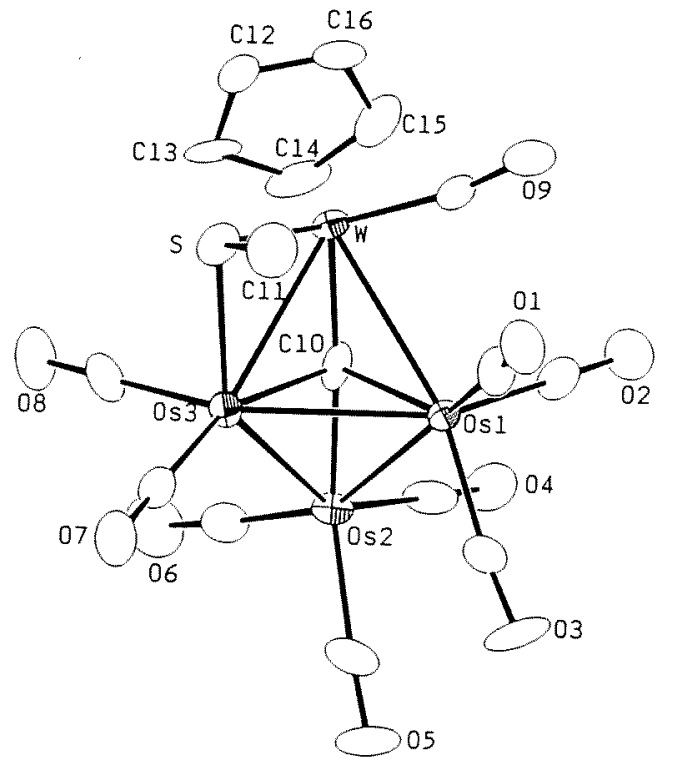

Fig. 3. Molecular structure of 4 and the atomic numbering scheme.

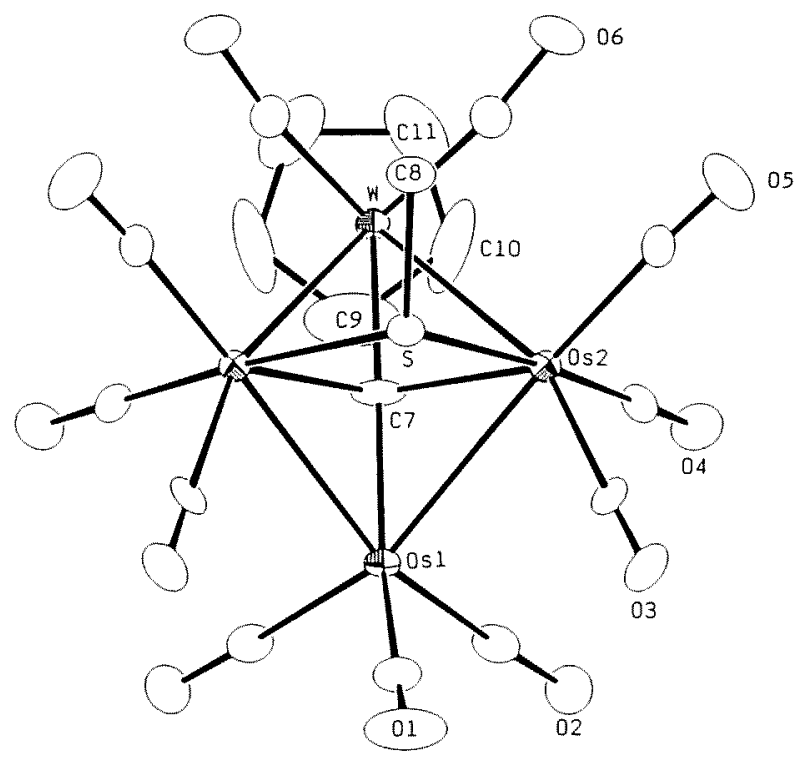

Fig. 4. Molecular structure of 5 and the atomic numbering scheme. 
is found to coordinate to two different $\operatorname{Os}(2)$ atoms, which are related to each other via reflection with distances $\mathrm{Os}(2)-\mathrm{S}=2.435(4) \AA$ and $\mathrm{Os}(2)-\mathrm{Os}(2)=3.490(2) \AA$ and angle $\angle \mathrm{Os}(2)-\mathrm{S}-\mathrm{Os}(2)=91.5(2)^{\circ}$. The methyl substitutent $C(8)$, which is tilted toward the unique $W$ atom, is found to site at a position trans to the $\mathrm{Cp}$ ligand and is eclipsed to the $\mathrm{CO}$ ligands on the $\mathrm{W}$ atom. Finally, assuming that the carbido and thiolate fragments each provides four and three electrons to the cluster, this molecule contains 64 valence electrons, which agree with the electron counting of tetranuclear complexes having four metal-metal bonds.

Although the structural characterization of $\mathbf{5}$ showed a highly symmetrical structure in the solid state, the variable temperature ${ }^{1} \mathrm{H}$ NMR studies indicated the existence of three isomers in solution. The ${ }^{1} \mathrm{H}$ NMR spectrum measured at $218 \mathrm{~K}$ exhibited three $\mathrm{Cp}$ signals at $\delta 5.81,5.47$, and 5.40 in the ratio $1: 2: 3$, and the respective $\mu$-SMe signals at $\delta 1.83,1.89$, and 1.91. Upon raising the temperature to $297 \mathrm{~K}$, we observed that the two downfield $\mathrm{Cp}$ resonances merged and coalesced into a broad signal at $\delta 5.60$, while the corresponding highfield $\mu$-SMe resonances merged to form a sharp signal at $\delta 1.89$ (Fig. 5).

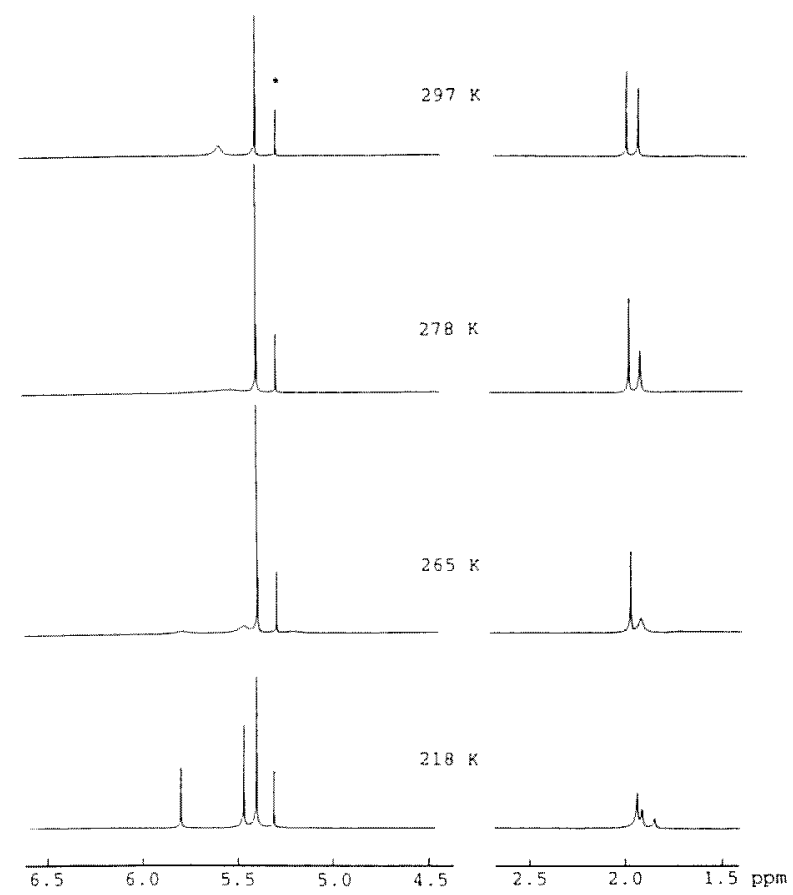

Fig. 5. Variable temperature ${ }^{1} \mathrm{H} N \mathrm{NMR}$ spectra of 5 in $\mathrm{CD}_{2} \mathrm{Cl}_{2}$ solution. 
This behavior indicated that the lirst two isomers undergo fast equilibrium in solution at room temperature, while the exchange with the third isomer is much slower under the conditions examined. The structural relationship of these three isomers is unclear at present; however, according to the X-ray structure mentioned previously, we propose that the isomerization is caused by moving the bridging thiolate ligand between three different positions with respect to the carbido carbon and two nonbridged metal atoms. As indicated in Scheme 2, the first isomer (A) contains a thiolate ligand which is sitting on the plane defined by the carbido carbon and two bridged Os atoms, whereas the other two isomers (B) and (C) possess a related structure in which the thiolate ligand is either parallel to the $\mathrm{WOs}_{2}$ plane or the second $\mathrm{Os}_{3}$ plane. Finally, the identities of these carbido clusters was verified by the ${ }^{13} \mathrm{C}$ NMR spectrum measured at $230 \mathrm{~K}$, which clearly exhibited three characteristic $\mu_{4}$ - C resonances at $\delta 329.9$, 329.7 , and 316.1 and three $\mathrm{W}-\mathrm{CO}$ signals at $\delta 224.0\left(J_{W-C}=161 \mathrm{~Hz}\right), 219.4$ $\left(J_{W-C}=161 \mathrm{~Hz}\right)$, and $208.0\left(J_{W-C}=162 \mathrm{~Hz}\right)$.

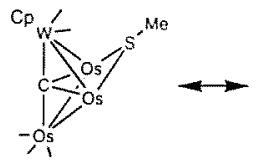

(C)

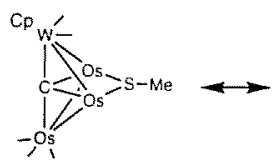

(A)

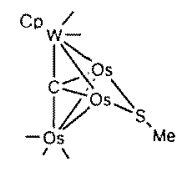

(B)

Scheme 2

\section{DISCUSSION}

Condensation of $\mathrm{Os}_{3}(\mathrm{CO})_{10}(\mathrm{NCMe})_{2}$ and $\mathrm{CpW}(\mathrm{CO})_{3}\left(\mathrm{CH}_{2} \mathrm{SMe}\right)$ produced three cluster compounds 1,2 , and 3 . The first cluster is a homonuclear cluster, while the other two $\mathrm{WOs}_{3}$ compounds are produced from the $1: 1$ combination of the starting materials. The diversified structures of these three compounds indicated a poor selectivity of this synthetic approach. The formation of 1 probably involves the coordination of sulfur atom to the $\mathrm{Os}_{3}(\mathrm{CO})_{10}$ framework followed by removal of $\mathrm{CpW}(\mathrm{CO})_{3}\left(\mathrm{CH}_{2}\right)$ fragment. The source of the hydride is possibly derived from the methylene group of $\mathrm{CpW}(\mathrm{CO})_{3}\left(\mathrm{CH}_{2} \mathrm{SMe}\right)$, but no study was made to verify this speculation. The second possibility involves the formation of trace amount of $\mathrm{MeSH}$ from decomposition of $\mathrm{CpW}(\mathrm{CO})_{3}\left(\mathrm{CH}_{2} \mathrm{SMe}\right)$, which then react with $\mathrm{Os}_{3}(\mathrm{CO})_{10}(\mathrm{NCMe})_{2}$ to afford the isolated 1.

For complex 2, we speculate that it is formed via a combined process of sulfur coodination to $\mathrm{Os}_{3}(\mathrm{CO})_{10}(\mathrm{NCMe})_{2}$ and capture of one additional $\mathrm{CO}$ ligand to afford the observed $\mathrm{Os}_{3}(\mathrm{CO})_{11}$ core. Attempts to 
improve the synthesis of $\mathbf{2}$ by employing the monoacetonitrile complex $\mathrm{Os}_{3}(\mathrm{CO})_{11}(\mathrm{NCMe})$ has not been successful. Attempts to use 2 as a precursor to other thiolate transition-metal clusters, which involved thermolysis in various common organic solvents and treatment with $\mathrm{Me}_{3} \mathrm{NO}$ reagent to induce the prior $\mathrm{CO}$ elimination, have also been frustratingly unsuccessful.

On the other hand, the most interesting and thoroughly studied compound is the methylene-thiolate derivative 3 , which is the only cluster compound containing a direct $\mathrm{W}-\mathrm{O}$ bond, and a bridging methylene and a bridging thiolate ligand attached to this $\mathrm{W}-\mathrm{O}$ s bond. To test that the $\mathrm{S}$ atom is required to coordinate to the $\mathrm{W}$ atom before it would induce the cleavage of $\mathrm{C}-\mathrm{S}$ bond, we carried out the reaction of $\mathrm{Os}_{3}(\mathrm{CO})_{10}(\mathrm{NCMe})_{2}$ and the decarbonylated $\mathrm{CpW}(\mathrm{CO})_{2}\left(\eta^{2}-\mathrm{CH}_{2} \mathrm{SMe}\right)$, as the latter possesses a cyclic $\mathrm{W}-\mathrm{CH}_{2}-\mathrm{S}$ interaction. After the coordination of $\mathrm{CpW}(\mathrm{CO})_{2}\left(\eta^{2}-\mathrm{CH}_{2} \mathrm{SMe}\right)$ to $\mathrm{Os}_{3}$ metal triangle, it would result in the formation of an intermediate with the $\mathrm{S}$ atom simultaneously bonded to both $\mathrm{W}$ and $\mathrm{Os}$ atoms, thus enhanced the yield for 3 . However, the products distribution of this reference reaction showed no significant difference from that reported earlier for $\mathrm{CpW}(\mathrm{CO})_{3}$ $\left(\mathrm{CH}_{2} \mathrm{SMe}\right)$ reaction. At this point, one could argue that the pre-coordination to $\mathrm{W}$ atom is of no importance in promoting the formation of 3 .

The spiked-triangular methylene cluster 3 can be converted to carbido clusters 4 and 5 through activation of the methylene group, as the extra $\mathrm{W}-\mathrm{Os}$ bonding interaction observed in $\mathbf{3}$ provided good stability against the cluster degradation. The carbido cluster $\mathbf{4}$ is a tetranuclear butterfly cluster containing $10 \mathrm{CO}$ and two bridging hydride ligands, while complex 5 contains 64 electrons and possesses $12 \mathrm{CO}$ ligands. Limited chemistry of these carbido clusters has been developed. Heating of 4 under $1 \mathrm{~atm}$ of $\mathrm{CO}$ in toluene failed to produce $\mathbf{5}$, but gave severe decomposition upon prolonged refluxing. In contrast, hydrogen reacted with $\mathbf{5}$ in toluene solution $\left(1 \mathrm{~atm}, 110^{\circ} \mathrm{C}, 5 \mathrm{hr}\right)$ to afford 4 in $12 \%$ yield and starting material $(70 \%)$. In view of these results, it is appropriate to say that the initial formation of carbido clusters $\mathbf{4}$ and $\mathbf{5}$ from methylene complex 3 proceeded through two different reaction channels: generation of $\mathbf{4}$ involves loss of three $\mathrm{CO}$ ligands, while the complex 5 is formed via competitive $\mathrm{H}_{2}$ elimination, without any accompanying $\mathrm{CO}$ elimination.

Finally, in summary, the condensation reaction reported in this article provides examples for the preparation of heterometallic thiolate clusters, and example for the conversion of methylene to carbido ligand. Despite that the chemistry observed for these compounds is very interesting, the poor thermal stability of $\mathrm{CpW}(\mathrm{CO})_{3}\left(\mathrm{CH}_{2} \mathrm{SMe}\right)$ and the low yields for the isolated cluster products seriously restrict the utilization of this thiolate reagent as the building blocks for cluster condensation. 


\section{SUPPLEMENTARY MATERIAL AVAILABLE}

A complete listing of thermal parameters, tables of nonessential bond distances, hydrogen atom coordinates, and listings of the observed and calculated structural factors for complexes $2,3, \mathbf{4}$, and $\mathbf{5}$ are available from one of the authors (Y.C.).

\section{ACKNOWLEDGMENTS}

We thank the National Science Council of Republic of China under Grant NSC 84-2113-M007-020 for financial support.

\section{REFERENCES}

1. (a) D. A. Roberts and G. L. Geofroy, in G. Wilkinson, F. G. A. Stone, and E. W. Abel (eds.), Comprehensive Organometallic Chemistry, Vol. 6, Chap. 40 (Pergamon, Oxford, 1982); (b) R. D. Adams, in D. F. Shriver. H. D. Kaesz, and R. D. Adams (eds.). The Chemistry of Metal Chusters, Chap. 3 (VCH, New York, 1990).

2. (a) Y. Chi, G.-H. Lee, S.-M. Peng, and C.-H. Wu (1989). Organometallics 8, 1574; (b) J.-H. Gong, C.-C. Chen, Y. Chi, S.-L. Wang, and F.-L. Liao (1993), J. Chem. Soc., Datton Trans. 1829; (c) J.-H. Gong, D.-K. Hwang, C.-W. Tsay, Y. Chi, S.-M. Peng, and G.-H. Lee (1994). Organometallics 13, 1720 .

3. (a) Y. Chi. F.-J. Wu. B.-J. Liu, C.-C. Wang, and S.-L. Wang (1989). J. Chem. Soc., Chem. Commun. 873; (b) D.-K. Hwang. Y. Chi, S.-M. Peng, and G.-H. Lee (1990). Organometallics 9. 2709; (c) Y. Chi (1992). J. Chin. Chem. Soc, 39, 591; (d) Y. Chi, C.-J. Su, L. J. Farrugia, S.-M. Peng, and G.-H. Lee (1994). Organometallica 13, 4167.

4. (a) Y. Chi, S.-H. Chuang. B.-F. Chen. S.-M. Peng, and G.-H. Lee (1990). J. Chem. Soc., Dalion Trans. 3033; (b) Y. Chi, S.-H. Chuang, L.-K. Liu, and Y.-S. Wen (1991), Organometallics 10, 2485

5. (a) Y. Chi, D.-K. Hwang, S.-F. Chen, and L.-K. Liu (1989). J. Chem. Soc. Chem. Commum. 1540; (b) Y. Chi, L.-K. Liu, G. Huttner, and L. Zsolnai (1990). J. Organomet. Chem. 390, C50.

6. (a) Y. Chi, R.-C. Lin, S.-M. Peng, and G.-H. Lee (1992). J. Chuster Sci. 3, 333; (b) R.-C. Lin. Y. Chi. S.-M. Peng, and G.-H. Lee (1992). lnorg. Chem. 31, 3818; (c) J.-C. Wang. R.-C. Lin, Y. Chi, S.-M. Peng, and G.-H. Lee (1993). Organometallics 12, 4061.

7. C.-W. Tsay, W.-C. Tu, Y. Chi, S.-M. Peng, and G.-H. Lee (1994). J. Chin. Chem. Soc. 41, 621.

8. R. B. King and M. B. Bisnette (1965). Lhorg. Chem. 4, 485.

9. (a) R. D. Adams (1985). Polyhedron 4, 2003; (b) E. J. Ditzel, M. P. Gómez-Sal, B. F. G. Johnson, J. Lewis, and P. R. Raithby (1987). J. Chem. Soc., Dalton Trans. 1623; (c) R. D. Adams, J. E. Babin, and M. Tasi (1986). Inorg. Chem. 25, 4514.

10. V. F. Allen, R. Mason, and P. B. Hitchoock (1977). J. Organomet. Chem. 140, 297.

11. M. R. Churchill and B. G. DeBoer (1977). Inorg. Chem. 16, 878 .

12. R. E. Benfield. B. F. G. Johnson, P. R. Raithby, and G. M. Sheldrick (1978). Acta Crystallogr. B34, 666.

13. E. J. Forbes, N. Goodhand, D. L. Jones, and T. A. Hamor (1979). J. Organomet. Chem. 182 , 143.

14. S.-M. Peng, G.-H. Lee, and Y. Chi (1990). Polyhedron 9, 1491. 
15. J. R. Shapley, A. C. Sievert, M. R. Churchill, and H. J. Wasserman (1981). J. Am. Chem. Soc. 103, 6975.

16. D. L. Davies, J. C. Jeffery, D. Miguel, P. Sherwood, and F. G. A. Stone (1987). J. Chem. Soc.. Chem. Commun. 454.

17. L. J. Farrugia, A. D. Miles, and F. G. A. Stone (1985). J. Chem. Soc., Dalton Trans. 2437.

18. D. L. Davies, J. C. Jeffery, D. Miguel, P. Sherwood, and F. G. A. Stone (1990). J. Organomet. Chem. 383, 463.

19. C.-H. Wu, Y. Chi, S.-M. Peng, and G.-H. Lee (1990), J. Chem. Soc., Dalion Trans. 3025.

20. (a) A. G. Cowie, B. F. G. Johnson, J. Lewis, and P. R. Raithby (1986). J. Organomet. Chem. 306, C63; (b) A. G. Cowie, B. F. G. Johnson, J. Lewis, and P. R. Raithby (1984). J. Chem. Soc., Chem. Commun. 1710; (c) J. S. Bradley, G. B. Ansell, M. E. Leonowicz, and E. W. Hill (1981). J. Am. Chem. Soc. 103, 4968. 